\title{
Hamiltonian dynamics of breathers with third-order dispersion
}

\author{
Shayan Mookherjea* and Amnon Yariv \\ Department of Applied Physics and Electrical Engineering, 128-95 California Institute of Technology, Pasadena, \\ California 91125
}

Received September 25, 2000; revised manuscript received March 20, 2001

\begin{abstract}
We present a nonperturbative analysis of certain dynamical aspects of breathers (dispersion-managed solitons) including the effects of third-order dispersion. The analysis highlights the similarities to and differences from the well-known analogous procedures for second-order dispersion. We discuss in detail the phase-space evolution of breathers in dispersion-managed systems in the presence of third-order dispersion. (C) 2001 Optical Society of America

OCIS codes: $190.5530,060.5530,190.4370$.
\end{abstract}

\section{INTRODUCTION}

Recent studies on the use of solitons in dispersionmanaged optical communications systems have shown that the effect of third-order dispersion, which arises from $d^{3} \beta / d \omega^{3}$, where $\beta(\omega)$ is the mode propagation constant at optical frequency $\omega$, may be significant since the average group-velocity dispersion can be much lower than the local group-velocity dispersion. In this paper we derive explicit analytical expressions for the dynamic behavior of solitonlike pulses (breathers) in such a system. Nonperturbative Lagrangian and Hamiltonian formulations have been useful in understanding the physical behavior of propagation in systems characterized by second-order dispersion alone. ${ }^{1}$ This research forms a counterpart to the perturbative analysis of Lakoba and Agrawal $^{2}$ based on a Hermite-Gaussian expansion of the pulse shape. The analysis presented here should also help understand the physical origins of certain features found in numerical simulations of the effects of third-order dispersion. ${ }^{3}$ Since the nonlinear (nonintegrable ${ }^{4}$ ) partial-differential (wave) equation is now of a higher order, it may be useful to show the applicability of a mathematical technique that is easily understood and widely used in a different problem.

We limit our attention to the steady-state (asymptotic) behavior of such pulses and therefore neglect the effect of continuum radiation on the dispersion-managed breather. $^{2}$ A soliton losing energy to linear dispersive waves in the normal-dispersion regime recoils further into the anomalous regime of the spectrum. ${ }^{5}$ This lowers the radiation amplitude, which arises as a consequence of the tail of the soliton spectrum in the normal-dispersion regime. It can then be expected that a quasi-stationary state is reached in which the radiation rate is so small that the soliton recoil is negligible, and we can ignore further radiation losses. Further, a practical communication system that uses dispersion management is likely to use optical filters and band-limited amplifiers, which can suppress out-of-band dispersive radiation and frequency shifts that are due to spectral recoil. ${ }^{6}$
Consequently, we are justified in assuming a symmetrical pulse shape. Given that we do not predict the generation of any new harmonics in this approximation, we would not expect any associated frequency shift; indeed, this will be shown to be a conserved quantity (as a generalized momentum canonically conjugate to a cyclic coordinate). It is interesting to note that the perturbative approach used by Lakoba and Agrawal $^{2}$ also does not predict any frequency shift for the lossless case considered here.

We quantify our results in terms of a representative hyperbolic secant pulse shape, but the method can be generalized to a number of different (localized) pulse shapes, e.g., Gaussian, triangular, etc. An appropriate Lagrangian is constructed for the nonlinear Schrödinger equation, and the condition of its extremality with respect to our pulse Ansatz forms variational equations of motion for the pulse parameters. ${ }^{7}$ Identifying generalized coordinates and their conjugate momenta leads to the Hamiltonian and a phase-plane analysis in terms of the acyclic coordinates. We highlight the modifications brought about by third-order dispersion to the well-known results of second-order dispersion-managed propagation.

The slowly varying normalized envelope $u(z, t)$ of an optical pulse in a lossless single-mode fiber with dispersion management in the group-velocity dispersion parameter and third-order dispersion follows the nonlinear Schrödinger equation, ${ }^{8}$

$$
i \frac{\partial u}{\partial z}+\frac{\sigma(z)}{2} \frac{\partial^{2} u}{\partial t^{2}}+|u|^{2} u-i \gamma \frac{\partial^{3} u}{\partial t^{3}}=0,
$$

with the standard notation. ${ }^{1,8}$ In this paper we consider a simple piecewise constant (step-function) dispersion map $\sigma(z)=\sigma_{ \pm}$, with $\sigma_{+}>0>\sigma_{-}$, and periodicity $\sigma(z)=\sigma\left(z+z_{T}\right)$.

\section{LAGRANGIAN FORMULATION AND PULSE ANSATZ}

The partial-differential equation (1) and its conjugate can be reduced to a system of ordinary differential equations 
by use of the averaged variational method, first introduced in the context of the evolution of solitons by Anderson. ${ }^{7}$ The motivation for this approach is well known, and we only point out that in the presence of third-order dispersion, the final term in Eq. (1) modifies the Euler-Lagrange equation,

$$
\begin{gathered}
\frac{\delta \mathcal{L}}{\delta u} \equiv \frac{\partial \mathcal{L}}{\partial u}-\frac{\partial}{\partial t} \frac{\partial \mathcal{L}}{\partial(\partial u / \partial t)}-\frac{\partial}{\partial z} \frac{\partial \mathcal{L}}{\partial(\partial u / \partial z)} \\
+\frac{\partial^{2}}{\partial t^{2}} \frac{\partial \mathcal{L}}{\partial\left(\partial^{2} u / \partial t^{2}\right)}=0
\end{gathered}
$$

and similarly for $u^{*}$ in place of $u$.

It may be easily verified, e.g., by direct substitution, that the following Lagrangian density (assumed to be at least $C^{1}$ in $u$ and its derivatives, except possibly on a set of measure zero) satisfies this condition,

$$
\begin{aligned}
\mathcal{L}\left(u, u^{*}\right)= & i\left(u \frac{\partial u^{*}}{\partial z}-u^{*} \frac{\partial u}{\partial z}\right)+\sigma(z)\left|\frac{\partial u}{\partial t}\right|^{2}-|u|^{4} \\
& -i \gamma\left(\frac{\partial^{2} u}{\partial t^{2}} \frac{\partial u^{*}}{\partial t}-\frac{\partial^{2} u^{*}}{\partial t^{2}} \frac{\partial u}{\partial t}\right)
\end{aligned}
$$

where a piecewise continuous $\sigma(z)$ satisfies the condition in parentheses.

The pulse shape we choose to base our analysis on should be capable of predicting pulse evolution, and therefore, in view of the term in Eq. (3) that contributes to third-order dispersion, we have to choose a pulse shape (envelope) somewhat more involved than for second-order dispersion alone. ${ }^{1}$ We take

$$
\begin{aligned}
u(z, t)= & A \sqrt{\eta} \operatorname{sech}[\eta(t-c)] \\
& \times \exp \left\{i\left[\omega(t-c)+\beta(t-c)^{2}+\frac{\phi}{2}\right]\right\},
\end{aligned}
$$

where $\eta, \beta, \omega, c$, and $\phi$ are free parameters that depend on $z$ and represent amplitude (and width), quadratic chirp, frequency shift from the carrier frequency (or velocity), pulse center in the moving reference frame (or groupvelocity variation in laboratory coordinates), and phase, respectively. In the interests of legibility we will not write out this dependence explicitly. Note that $\omega$ as used above is distinct from the optical frequency $\omega_{0}$. The pulse energy is then evaluated to

$$
\int_{-\infty}^{\infty} u(z, t) u(z, t)^{*} \mathrm{~d} t=2 A^{2}
$$

Similar forms of the envelope Ansatz have been used elsewhere $^{9}$ for analyzing pulse interactions in secondorder dispersion-managed systems and reflect the fact that the equations under consideration need more degrees of freedom than offered by simpler assumptions.

We now write the Lagrangian using Eqs. (3) and (4). A fair amount of algebra leads to the following expressions for the four terms on the right-hand side of Eq. (3), where we use the shorthand $\beta^{\prime} \equiv \mathrm{d} \beta / \mathrm{d} z$, etc.:

$$
\begin{aligned}
i\left(u \frac{\partial u^{*}}{\partial z}-u^{*} \frac{\partial u}{\partial z}\right) & \\
\rightarrow & 2\left[(t-c)^{2} \beta^{\prime}+(t-c)\right. \\
& \left.\times\left(\omega^{\prime}-2 \beta c^{\prime}\right)+\left(\phi^{\prime}-\omega c^{\prime}\right)\right] u u^{*}, \\
\left.|| \frac{\partial u}{\partial t}\right|^{2} \rightarrow & \sigma\left\{4(t-c)^{2} \beta^{2}+\eta^{2} \tanh [\eta(t-c)]^{2}\right. \\
& \left.+4(t-c) \beta \omega+\omega^{2}\right\} u u^{*}, \\
-i \gamma\left(\frac{\partial^{2} u}{\partial t^{2}} \frac{\partial u^{*}}{\partial t}-\frac{\partial^{2} u^{*}}{\partial t^{2}}\right. & \left.\frac{\partial u}{\partial t}\right) \\
\rightarrow & \gamma\left(16(t-c)^{3} \beta^{3}+24(t-c)^{2} \beta^{2} \omega\right. \\
& +2 \omega\left(\eta^{2}+\omega^{2}\right) \\
& -2 \beta\{\eta \tanh [\eta(t-c)] \\
& \left.\left.-(t-c)\left(\eta^{2}+3 \omega^{2}\right)\right\}\right) u u^{*} .
\end{aligned}
$$

In each of the above expressions we can drop those terms that are odd in $t$ as such terms do not contribute when multiplied by $u u^{*}$ and integrated over $t$. These include terms that are proportional to odd powers of $(t-c)$ and those that vary as $\tanh (t-c)$. After some simplification, we can write the Lagrangian as

$$
\begin{aligned}
L= & A^{2}\left\{\frac{\pi^{2}}{3 \eta^{2}} \frac{\mathrm{d} \beta}{\mathrm{d} z}+4\left(\frac{\mathrm{d} \phi}{\mathrm{d} z}-\omega \frac{\mathrm{d} c}{\mathrm{~d} z}\right)-4 A^{2} \frac{\eta}{3}\right. \\
& +2 \sigma\left[\omega^{2}+\frac{1}{3}\left(\frac{\pi^{2} \beta^{2}}{\eta^{2}}+\eta^{2}\right)\right] \\
& \left.+4 \gamma \omega\left(\frac{\pi^{2} \beta^{2}}{\eta^{2}}+\omega^{2}+\eta^{2}\right)\right\} .
\end{aligned}
$$

with a clearly nontrivial role for $\gamma$. The Lagrangian function (7) has no explicit $z$ dependence, and so the Hamiltonian is a constant of the motion. This will be the starting point of our discussion in the following section.

We note in passing that it is possible to construct a Lagrangian for pulse shapes other than the hyperbolic secant reminiscent of the Schrödinger soliton, generalizing to any localized Ansatz for which the amplitude and the pulse width are related in the same way as above.

\section{HAMILTONIAN DYNAMICS}

We apply the Legendre transformation ${ }^{10}$ to the Lagrangian (7) to generate the Hamiltonian. We take our canonical coordinates as $\beta$ and $c$ with conjugate momenta

$$
\begin{aligned}
& p_{\beta}=\frac{\partial}{\partial \beta^{\prime}} L=\frac{\pi^{2} A^{2}}{3 \eta^{2}}, \\
& p_{c}=\frac{\partial}{\partial c^{\prime}} L=-4 A^{2} \omega
\end{aligned}
$$

and express the Lagrangian in terms of these variables, substituting $p_{\beta}$ for $\eta$ and $p_{c}$ for $\omega$, 


$$
\begin{aligned}
L= & A^{2}\left[\gamma p_{c}\left(\frac{p_{c}^{2}}{16 A^{6}}+\frac{\pi^{2}}{3 p_{\beta}}+\frac{3 p_{\beta} \beta^{2}}{A^{4}}\right)\right. \\
& \left.+\sigma\left(\frac{2 \pi^{2} A^{2}}{9 p_{\beta}}+\frac{p_{c}^{2}}{8 A^{4}}+\frac{2 p_{\beta} \beta^{2}}{A^{2}}\right)-\frac{4 \pi A^{3}}{3 \sqrt{3 p_{\beta}}}+4 \frac{\mathrm{d} \phi}{\mathrm{d} z}\right] \\
& +p_{\beta} \beta^{\prime}+p_{c} c^{\prime} .
\end{aligned}
$$

[It can be verified after extensive algebra (symbolic manipulation tools such as Mathematica are useful) that $\operatorname{det}\left(\partial^{2} L / \partial p_{i} \partial p_{j}\right) \neq 0$.]

The Hamiltonian is

$$
\begin{aligned}
H(\beta, c ; \eta, \omega ; z)= & p_{\beta} \beta^{\prime}+p_{c} c^{\prime}-L \\
= & 2 A^{2}\left[-\left(2 \gamma \omega+\frac{\sigma}{3}\right)\left(\frac{\pi^{2} \beta^{2}}{\eta^{2}}+\eta^{2}+\omega^{2}\right)\right. \\
& \left.+\frac{2}{3}\left(A^{2} \eta-\omega^{2} \sigma\right)-2 \frac{\mathrm{d} \phi}{\mathrm{d} z}\right]
\end{aligned}
$$

where we need not explicitly consider the phase dependence $\phi(z)$ and its canonical momentum in the equations of motion as these terms drop out (indicating that the phase depends on the other parameters and plays no role in the dynamics ${ }^{11}$ ). For a conserved Hamiltonian, $\mathrm{d} H / \mathrm{d} z=0$ or $H=$ constant; thus we can construct level sets of $H$. We return to this in Section 4. Although such an analysis (similar to that carried out elsewhere for second-order dispersion ${ }^{1}$ ) can lead to a closed-form expression for $\eta(z)$, the results are extremely unwieldy and unlikely to be of much practical use.

Instead, we obtain the equations of motion from Eq. (10),

$$
\begin{aligned}
& \frac{\mathrm{d} \beta}{\mathrm{d} z}=-\frac{2}{\pi^{2}} A^{2} \eta^{3}-2(\sigma+6 \gamma \omega)\left(\beta^{2}-\frac{\eta^{4}}{\pi^{2}}\right), \\
& \frac{\mathrm{d} \eta}{\mathrm{d} z}=-2 \beta \eta(\sigma+6 \gamma \omega),
\end{aligned}
$$

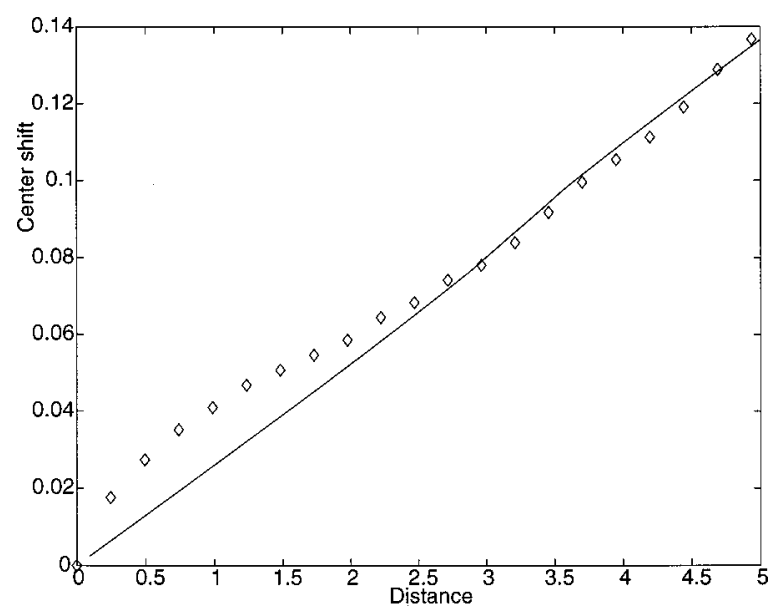

Fig. 1. Position of pulse center $c(t)$, normalized to the input pulsewidth, versus propagation distance $z$, normalized to the single-soliton period. The data points are from a split-step Fourier simulation, and the solid curve is from numerical integration of Eq. (12.1), which was derived from theoretical considerations.

$$
\begin{aligned}
& \frac{\mathrm{d} c}{\mathrm{~d} z}=\sigma \omega+\gamma\left(\frac{\pi^{2} \beta^{2}}{\eta^{2}}+\eta^{2}+3 \omega^{2}\right) \\
& \frac{\mathrm{d} \omega}{\mathrm{d} z}=0
\end{aligned}
$$

where we have used the fact that $\sigma(z)$ is piecewise constant with derivative zero on the entire domain of $z$ except for a set of measure zero. The last equation of the above set follows from the observation that $L$ does not contain the cyclic coordinate $c$ explicity; its associated momentum is then a constant of the motion.

The modification brought about by the third-order nonlinearity in the first two of the above set of equations is to alter the strength of the dispersion map. The third equation describes the drift in the center of the pulse (in the moving reference frame). Clearly there are terms in these equations that are explicity dependent on the thirdorder dispersion coefficient $\gamma$ and describe effects predicted only when third-order dispersion is taken into account. In Fig. 1 we show a comparison between our theory [Eq. (12.1)] and direct numerical simulation of the drift in the pulse center brought about by third-order dispersion. The close agreement for the higher ranges of $z$ (recall that our analysis is an asymptotic one) shows the robustness of the Hamiltonian approach, withstanding the presence of dispersive waves in the numerical simulation that we have to neglect in a Hamiltonian approach. We note that the peak amplitude does indeed move more or less linearly toward the positive time axis, associated with a change in the group velocity of the pulse, as was predicted from a purely numerical simulation in Ghafouri-Shiraz et al. ${ }^{3}$

\section{PHASE-PLANE DYNAMICS}

We use a phase-plane analysis similar to Kutz et al. ${ }^{1}$ to further understand the behavior of the system. Since $c$ is a cyclic coordinate, its conjugate momentum is a conserved quantity, and we plot the level sets of the Hamiltonian from Eq. (10) in the $(\eta, \beta)$ plane. Since Eq. (1) is autonomous (the independent variables do not appear explicitly), trajectories in phase space do not intersect. We take $\sigma(z)$ to be constant $=\sigma$ when we plot the analytical results, and we consider normal $(\sigma<0)$ and anomalous $(\sigma>0)$ dispersion separately. This corresponds to propagation in fibers of uniform second-order dispersion, and dispersion maps can be constructed by adding together such sections of appropriate length. We return to this important point below. As in the case of the model without third-order dispersion, the dynamics are markedly different in the two cases. As a mathematical aside, we can show that breathing solutions do exist (Appendix A).

The phase-plane plots for normal and anomalous dispersion are shown in Figs. 2 and 3. The mirror symmetry of both plots about the axes $\beta=0$ implies invariance of the solutions to Eqs. (11.1) and (11.2) under the transformations $t \rightarrow-t$ and $(\eta \rightarrow \eta, \beta \rightarrow-\beta$ ). For normal dispersion $(\sigma<0)$ the phase flow is clockwise, and all orbits limit at the origin as $z \rightarrow \pm \infty$. In contrast, for anomalous dispersion $(\sigma>0)$ the counterclockwise 


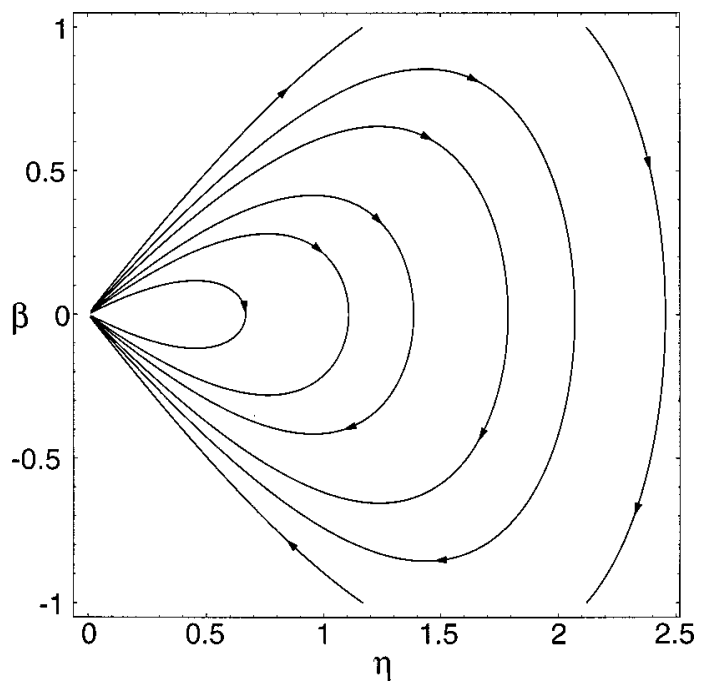

Fig. 2. Phase portraits for normal dispersion: level sets of the Hamiltonian in the $(\eta, \beta)$ plane with $\sigma=-1$. Note that all trajectories start and end at the origin.

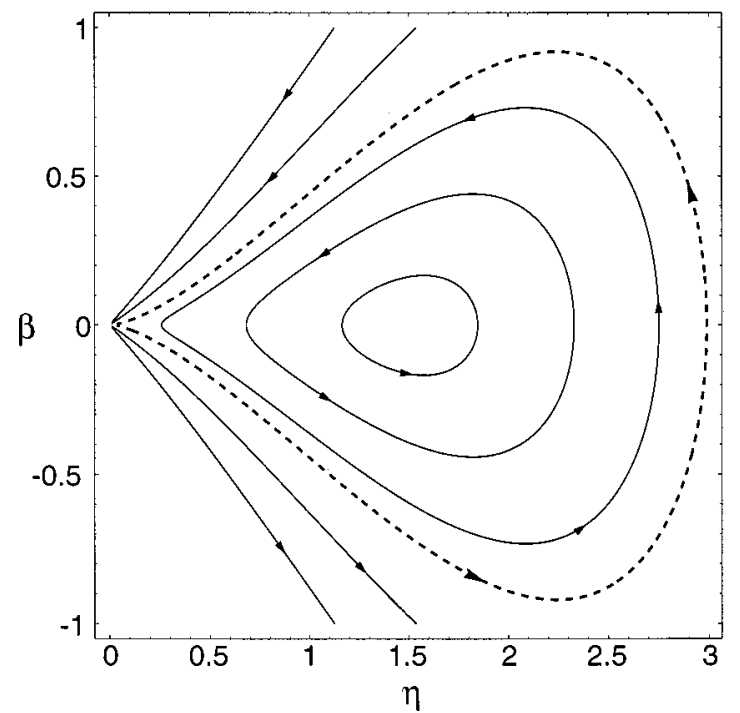

Fig. 3. Phase portraits for anomalous dispersion: level sets of the Hamiltonian in the $(\eta, \beta)$ plane with $\sigma=1$. Trajectories within the separatrix, shown by the dotted curve, are periodic.

phase flow indicates not only homoclinic orbits to the origin, as in the previous case, but also periodic solutions inside the separatrix.

The separatrix defines the boundary between solutions that are periodic and those that flow into the origin. Its equation for dispersion-managed systems with thirdorder dispersion can be obtained from Eq. (10) by setting $\beta=0$ and solving for the Hamiltonian value $H_{0}$ such that the origin is part of the solution. The result is

$$
\beta= \pm \frac{\eta}{\pi}\left(\frac{2}{3} \frac{A^{2} \eta}{\sigma / 3+2 \gamma \omega}-\eta^{2}\right)^{1 / 2} .
$$

Further, we note that for the case of anomalous dispersion the width (in $\beta$ ) of the separatrix at its widest in the $(\eta, \beta)$ space is given by

$$
\delta \beta=\frac{2 \pi}{\sqrt{3}}\left[\frac{A^{2}}{2 \pi(\sigma / 3+2 \gamma \omega)}\right]^{2},
$$

which shows that third-order dispersion quadratically lowers the phase-space domain over which periodic solutions exist. The corresponding maximum swing in the quadratic chirp is, of course, smaller. This analytical result is of importance in designing communication systems and protocols that must have available estimates for the effects of third-order dispersion in the evolution of the pulse width/chirp with propagation distance. We can, in principle, compensate for the increase of the denominator for $\gamma \neq 0$ by increasing $A$. Viewed from a different perspective, breathers with third-order dispersion that maintain the same width in phase space as defined by Eq. (14) have greater energy, leading to lower error rates in typical communications systems.

We can use Figs. 2 and 3 to construct a dispersion map, similar to the procedure carried out in Kutz et al. ${ }^{1}$ Figure 4 shows a superposition of phase-space orbits from propagation along uniform normal-dispersion (ND) and anomalous-dispersion (AD) fibers, with two of the many possible dispersion-mapped orbits identified, each starting for example with an unchirped pulse at the $\beta=0$ line. First we encounter (clockwise) propagation along a certain length $z_{1} / 2$ of ND fiber followed by (counterclockwise) propagation along a length $z_{2}$ of $\mathrm{AD}$ fiber. Finally, to symmetrize the map, we require propagation along length $z_{1} / 2$ of the same normal-dispersive fiber as before. The breather traverses this map in the direction of the arrows. The transitions between $\mathrm{ND}$ and $\mathrm{AD}$ fiber are marked with circles: we clearly are free to pick contours, e.g., so that the pulse amplitude (width) satisfies certain limits imposed by a systems-design criterion. More complicated dispersion maps can be very easily constructed simply by tracing out the trajectory of the breather and ensuring that we return to the initial point after some distance that then defines the period. As mentioned earlier, orbits in phase space do not intersect one another, so the

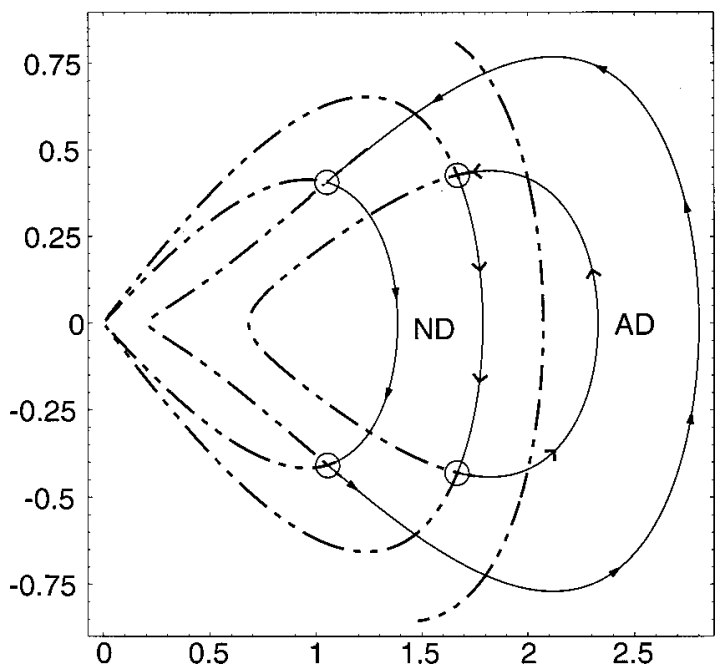

Fig. 4. Construction of dispersion maps based on Figs. 2 and 3. Transitions between the two types of fiber are indicated with circles. Two possible trajectories traversed by a breather are indicated by arrows. 


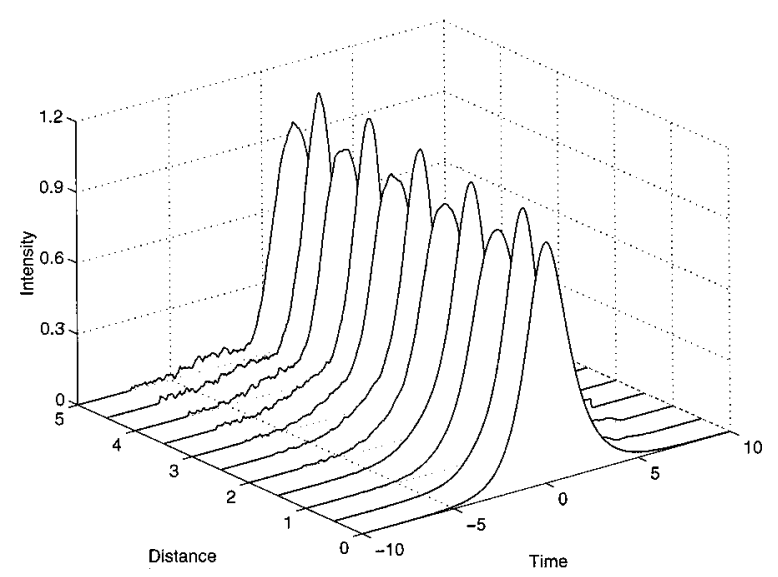

Fig. 5. Numerical simulation of pulse propagation over several periods along a dispersion map constructed obtained by Fig. 4 .

evolution of a breather along a trajectory we construct is necessarily deterministic and unique.

Propagation along such a symmetric map composed of two types of fiber with lengths $L_{1}$ and $L_{2}$ and groupvelocity dispersion coefficients $\beta_{1}^{\prime \prime}$ and $\beta_{2}^{\prime \prime}$, respectively, is shown in Fig. 5. The map is defined by the following normalized relationships:

$$
\frac{\beta_{2}^{\prime \prime}(z)}{\beta_{1}^{\prime \prime}(z)}=\frac{-5.5}{+1}, \quad \frac{L_{2}}{L_{1}+L_{2}}=\frac{0.2}{0.8+0.2} .
$$

The slight asymmetry and dispersive tails are ignored in the above Hamiltonian analysis and could conceivably be compensated for to a certain extent by optical filtering. ${ }^{12}$ Nevertheless, the breathing nature of the pulse is clearly visible.

In conclusion, we have analyzed the nonlinear Schrödinger equation accounting for third-order dispersion using a nonperturbative variational approach. A Lagrangian was constructed from the modified Euler-Lagrange equation, and identification of appropriate generalized coordinates and their conjugate momenta leads to a Hamiltonian formulation of the pulse dynamics. Using a hyperbolic secant Ansatz and allowing for quadratic chirp as well as shifts in the group velocity, we have formed equations of motion of the principal pulse parameters, and we have shown by a phase-plane analysis that breathers can be formed in the anomalous-dispersion regime. The presence of third-order dispersion reduces the relevant ( $\eta$, $\beta$ ) parameter space over which such periodic solutions exist; conversely, breathers with third-order dispersion have enhanced energy that can be of benefit in optical communications. We have shown how to compute dispersion maps for systems that include third-order dispersion, and we have indicated their agreement with numerical simulations. The results are particularly useful in the design of dispersion-managed communication systems, with low average second-order dispersion.

\section{APPENDIX A: EXISTENCE OF BREATHING SOLUTIONS FOR THIRD-ORDER DISPERSION}

It may be useful to show mathematically that breathing solutions can exist even with third-order dispersion. We carry out a linearized stability analysis, and we begin by determining the fixed points of the system by setting the left-hand sides of Eq. (11) to zero. There are two solutions:

$$
\begin{aligned}
& \text { (I) } \beta=0, \eta=0, \\
& \text { (II) } \beta=0, \quad \eta=\frac{A^{2}}{\sigma+6 \gamma \Omega} \equiv \eta_{0} .
\end{aligned}
$$

The first fixed point is (triply) degenerate and cannot be used in linear-stability theory. We can say, however, that the origin cannot be a center in the phase plane: orbits approaching a center point tend to oscillate around it, and negative amplitudes are not physically meaningful. The solutions that involve this fixed point are homoclinic orbits, i.e., they approach one and the same fixed point as $z \rightarrow \pm \infty$.

With regard to the second fixed point, we take small perturbations $(\Delta \eta, \Delta \beta)$ around the fixed point, and linearizing the equations, after some algebra,

$$
\begin{aligned}
& \Delta \eta^{\prime}=-2 A^{2} \Delta \beta, \\
& \Delta \beta^{\prime}=\frac{2}{\pi^{2}} A^{2} \eta_{0}^{2} \Delta \eta,
\end{aligned}
$$

which leads to the eigenvalues

$$
\lambda_{ \pm}= \pm i \frac{2}{\pi} \eta_{0}^{2}(\sigma+6 \gamma \omega)= \pm i \frac{2}{\pi} \frac{A^{4}}{\sigma+6 \gamma \omega}
$$

and eigenvectors

$$
\mathbf{v}_{ \pm}=\left( \pm i \frac{\pi}{\eta+0}, 1\right) .
$$

Since both the eigenvalues are purely imaginary, the point $\left(\eta_{0}, 0\right)$ is a center in the phase plane ${ }^{13}$ that is physically satisfying for a model without dissipation. Furthermore, for constant $\sigma(z)=\sigma_{ \pm}$, since from Eq. (11)

$$
\boldsymbol{\nabla} \cdot(\mathrm{d} \eta / \mathrm{d} z, \mathrm{~d} \beta / \mathrm{d} z)=-6 \beta\left(\sigma_{ \pm}+6 \gamma \omega\right)
$$

changes in sign in the range of $\beta$ that we consider, we expect that the above (autonomous) system of equations can have periodic solutions according to the Bendixson criterion. $^{13}$ Moreover, for the case of anomalous dispersion these periodic solutions can be physically interpreted as breathers similar to the case of the model without third-order dispersion. ${ }^{1}$

\section{ACKNOWLEDGMENT}

This study was supported by the Office of Naval Research and the U.S. Air Force Office of Scientific Research. We thank reviewers for valuable comments.

*E-mail address: shayan@caltech.edu.

\section{REFERENCES}

1. J. N. Kutz, P. Holmes, S. G. Evangelides, and J. P. Gordon, "Hamiltonian dynamics of dispersion managed breathers," J. Opt. Soc. Am. B 15, 87-96 (1998).

2. T. I. Lakoba and G. P. Agrawal, "Effects of third-order dis- 
persion on dispersion-managed solitons," J. Opt. Soc. Am. B 16, 1332-1343 (1999).

3. H. Ghafouri-Shiraz, P. Shum, and M. Nagata, "A novel method for analysis of soliton propagation in optical fibers," IEEE J. Quantum Electron. 31, 190-200 (1995).

4. T. I. Lakoba, "Non-integrability of equations governing pulse propagation in dispersion-managed optical fibers," Phys. Lett. A 260, 68-77 (1999).

5. N. Akhmediev and M. Karlsson, "Cherenkov radiation emited by solitons in optical fibers," Phys. Rev. A 51, 26022607 (1995).

6. I. M. Uzunov, M. Gölles, and F. Lederer, "Soliton interaction near the zero-dispersion wavelength," Phys. Rev. E 52, 1059-1071 (1995).

7. D. Anderson, "Variational approach to nonlinear pulse propagation in optical fibers," Phys. Rev. A 27, 3135-3145 (1983).
8. G. P. Agrawal, Nonlinear Fiber Optics (Academic, New York, 1989).

9. T. Inoue, H. Suguhara, A. Maruta, and Y. Kodama, "Interactions between dispersion-managed solitons in optical time-division-multiplexed system," IEEE Photonics Technol. Lett. 12, 299-301 (2000).

10. F. A. Scheck, Mechanics: From Newton's Laws to Deterministic Chaos, 3rd. ed. (Springer, New York, 1999).

11. P. Holmes and J. N. Kutz, "Dynamics and bifurcations of a planar map modeling dispersion managed breathers," SIAM (Soc. Ind. Appl. Math.) J. Appl. Math. 59, 1288-1302 (1999).

12. J. Kurnasako, M. Matsumoto, and S. Waiyapot, "Linear stability analysis of dispersion-managed solitons controlled by filters," J. Lightwave Technol. 18, 1064-1068 (2000).

13. F. Verhulst, Nonlinear Differential Equations and Dynamical Systems (Springer, New York, 2000). 